\title{
Understanding the Structure and Function of the Immunological Synapse
}

\author{
Michael L. Dustin ${ }^{1}$, Arup K. Chakraborty², and Andrey S. Shaw ${ }^{3}$ \\ ${ }^{1}$ Program in Molecular Pathogenesis, Skirball Institute of Biomolecular Medicine and Department of Pathology, \\ New York University, New York, New York 10016 \\ ${ }^{2}$ Department of Chemical Engineering, Department of Chemistry, Division of Biological Engineering, \\ Massachusetts Institute of Technology, Cambridge, Massachusetts 02139 \\ ${ }^{3}$ Department of Pathology and Immunology and Howard Hughes Medical Institute, Washington University \\ School of Medicine, St. Louis, Missouri 63110 \\ Correspondence: shaw@pathology.wustl.edu
}

The immunological synapse has been an area of very active scientific interest over the last decade. Surprisingly, much about the synapse remains unknown or is controversial. Here we review some of these current issues in the field: how the synapse is defined, its potential role in T-cell function, and our current understanding about how the synapse is formed.

$T_{t}$ cells are activated when they recognize peptide-MHC complexes on the surface of antigen presenting cells (APC) (Babbitt et al. 1985). But the exact process regarding how antigenic pMHC complexes are recognized and transduced into signals is still incompletely understood. Naïve T cells enter secondary lymphoid organs such as the lymph node and scan dendritic cells for the presence of rare specific pMHC complexes (Miller et al. 2004). After recognizing less than 10 specific $\mathrm{pMHC}$ complexes, naïve $\mathrm{T}$ cells maintain long contacts $(6-18 \mathrm{~h})$ with dendritic cells before being committed to enter cell cycle and differentiate into effector T cells (Iezzi et al. 1998; Irvine et al. 2002; Mempel et al. 2004).

The immunological synapse (IS) refers to the organization of membrane proteins that occurs at the interface between the $\mathrm{T}$ cell and the
APC during these long contacts and also during the effector phase (Grakoui et al. 1999; Monks et al. 1998). Interest in studying the IS stems from ideas that the supramolecular structures that form at the IS underlies the high sensitivity of $\mathrm{T}$ cell recognition and that understanding these structures will lead to better insights into how antigen recognition leads to the decision of a $\mathrm{T}$ cell to proliferate, differentiate, and function.

Springer first put forward the concept that receptors would segregate laterally during cell interactions (Springer 1990). Subsequently, Kupfer was the first to show that proteins in the contact area between a T cell and APC segregate laterally (Monks et al. 1998). Specifically, he noted that the integrin, LFA-1, became concentrated in an outer ring, known as the peripheral supramolecular activation complex ( pSMAC) and the TCR became concentrated in the center,

Editors: Lawrence E. Samelson and Andrey Shaw

Additional Perspectives on Immunoreceptor Signaling available at www.cshperspectives.org

Copyright (C) 2010 Cold Spring Harbor Laboratory Press; all rights reserved; doi: 10.1101/cshperspect.a002311

Cite this article as Cold Spring Harb Perspect Biol 2010;2:a002311 
in a zone known as the central supramolecular activation complex (cSMAC) (Monks et al. 1998)(Fig. 1). We showed that CD2 could segregate from LFA-1 and concentrate in the center of a hybrid cell-planar bilayer junction and suggested that these patterns and those described by Monks et al. (1998) provided evidence for the previously hypothesized immunological synapse (Dustin et al. 1998; Norcross 1984). The function of this receptor segregation is still not completely understood but it was initially hypothesized that formation of this pattern might be related to T-cell activation and constitute a "molecular machine" that would be formed in response to the presence of antigenic ligand and that this "molecular machine" might function to sustain signaling for long periods of time and direct subsequent T-cell differentiation (Grakoui et al. 1999).

\section{FUNCTIONS OF THE IMMUNOLOGICAL SYNAPSE}

Over the last 12 years, intensive research into the function of the IS implicates the IS in two major categories of functions: 1) priming of responses

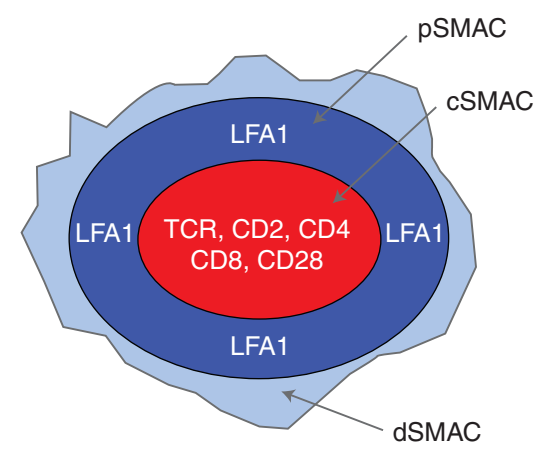

in which the $\mathrm{T}$ cell is receiving information and 2) effector functions in which the $\mathrm{T}$ cell is sending information. For example, the pSMAC has been suggested to function for cytolytic $\mathrm{CD} 8+$ cells as a sealing ring, preventing the leakage of cytolytic granule contents to bystander cells (Stinchcombe et al. 2006). Much work also implicates the cSMAC as a site for receptor internalization and degradation, which is important for regulation of longer-term responses of the T cell (Lee et al. 2002; Lee et al. 2003; Varma et al. 2006). There is evidence that the ubiquitination machinery is concentrated in the CSMAC and that formation of the CSMAC is required for receptor degradation (Lee et al. 2003; Lee et al. 2002; Wiedemann et al. 2005). Lastly, the application of highly sensitive microscopy techniques has revealed that sustained signaling in a mature IS is mediated by TCRs in small clusters of 5-30 molecules and that these clusters move centripetally toward the cSMAC (Varma et al. 2006; Yokosuka et al. 2005). In some cases the signaling elements assembled by the TCR display centripetal motion after separating from the TCR (Bunnell et al. 2002).

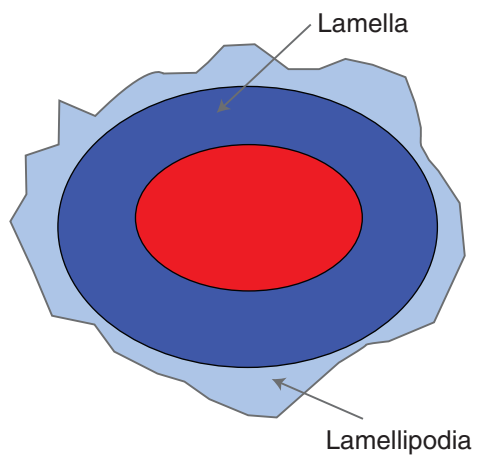

Figure 1. Structure of the immunological synapse. The basic structure of the "organized" immunological synapse with SMACs is shown (left). In the center is the central supramolecular activation complex or cSMAC, which contains receptors like the TCR, CD28, CD4, CD8, and CD2. Newer studies suggest that the cSMAC may be divided into an outer area containing CD28 and an inner area containing the TCR (not shown). The ring that surrounds the CSMAC is called the peripheral supramolecular activation complex or pSMAC. This domain is mainly populated by the integrin molecule LFA-1. Outside of the pSMAC is another domain known as the distal supramolecular activation complex. Originally the dSMAC was thought not be important and contain all of the molecules that are not specifically recruited to the cSMAC or pSMAC but it is increasingly becoming appreciated that the dSMAC is an area of active membrane movement. This suggests that the pSMAC and dSMAC may be analogous to the actin structures known as the lamellae and lamellipodia, respectively (right). 
There are many situations in which mature ISs with pSMACs and cSMACs are not observed in vitro and it remains an open question how common these structures are in vivo (Barcia et al. 2006; Brossard et al. 2005; McGavern et al. 2002; Purtic et al. 2005; Reichert et al. 2001; Tseng et al. 2008). Mature IS formation is most readily observed with $B$ cell tumors, resting $B$ cells, and supported planar bilayers and is dependent on antigen quality (Grakoui et al. 1999; Monks et al. 1998). Furthermore, the size of the cSMAC is linearly dependent on antigen quantity in the supported planar bilayer system in which formation can be most sensitively tracked (Grakoui et al. 1999; Varma et al. 2006). The frequency of mature IS visualized during priming of naïve $\mathrm{T}$ cells is typically less than $50 \%$ at any time, but this may, in part, reflect the periodic symmetry breaking and migration that is interspersed with stable IS formation (Sims et al. 2007). Reflecting its potential importance in cell killing, effector CD8 T cells form synapses very efficiently with $\sim 80 \%$ forming mature synapses on lipid bilayers (Beal et al. 2008). The applicability of lessons from the mature IS models (e.g., $\mathrm{B}=$ cell tumors and planar bilayers) to situations in which such highly ordered structures do not appear to form (e.g., priming of naïve T cells by DC) is a matter of controversy.

In this review, we examine current thoughts about how synapses form and the function of the IS. Advanced imaging methods have led to highly detailed images that have given new insight into how synapses form and what the basic parameters are in vivo. Computational approaches have helped us to understand what is happening at the IS and what effects synapse formation will have on T-cell activation events.

\section{GETTING LYMPHOCYTES TO STOP!}

Lymphocytes are constantly moving through the body in search of antigen. When they finally recognize antigen, an important step is they must stop and form a stable contact with the antigen-presenting cell. The antigen-induced up-regulation of LFA-1 binding to ICAM-1 to generate a strong adhesive surface is a critical step in slowing cell motility (Dustin and Springer 1989; Dustin et al. 1997; Scholer et al. 2008). Although this is probably the first step in the process of immunological synapse formation, adhesion strengthening itself is not sufficient.

Early experiments using different in vitro models made strikingly different predictions about what might occur in vivo. Methods in which $\mathrm{T}$ cells encountered antigen in the absence of extracellular matrix showed antigen stop signals (Dustin et al. 1997; Grakoui et al. 1999; Negulescu et al. 1996), whereas methods in which T cells encounter dendritic cells in collagen gels showed continual motility (Gunzer et al. 2000). This controversy was resolved by in vivo imaging that consistently showed $\mathrm{T}$ cell arrest with antigen presenting dendritic cells, but also showed that the time from first antigen recognition to arrest was variable and related to the quantity of antigen present; more antigen resulted in the faster initiation of stable contacts (Henrickson et al. 2008; Mempel et al. 2004). The duration of the stable T-DC interactions in vivo is on the order of $1 \mathrm{~d}$ and activation of T cells may involve multiple cycles of stable interactions during the proliferative phase of the response (Celli et al. 2007).

Signaling through the TCR stimulates calcium entry and calcium is likely to play a key role in mediating the stop signal (Skokos et al. 2007). Analysis of T-DC interactions in mice lacking ICAM-1 showed that the LFA-1-ICAM1 interaction is required for stable interactions and development of memory responses but is not required for cytokine production or proliferation of CD8 T cells (Scholer et al. 2008). This suggests that stable T-DC interactions may be important to induce the formation of immunological memory, but not required for other early aspects of T-cell activation.

\section{Front and Back: Repolarizing the Cell}

In addition to increased adhesion, the repolarization of the $\mathrm{T}$ cell is a critical event in generation of the immunological synapse. The first type of polarization occurs when the cells exit the blood and migrate into tissue. Chemokines are thought to be the initial trigger to prepare 
the cell for extravasation. Chemokine signaling through G-protein coupled receptors results in localized PIP3 production and subsequent polarization of the actin filament system (Hirsch et al. 2000; Li et al. 2000; Niggli and Keller 1997). Chemokines can be present in soluble gradients, but in lymphoid tissues, the chemokine is thought to decorate the surface of the reticular fiber network. Polarization of the migrating lymphocyte results in a protrusive leading forward edge, or lamellipodium, that is highly sensitive to the detection of antigen and a bulbous appendage at the rear, the uropod. (Sanchez-Madrid and Serrador 2009).

\section{POLARITY AND CELL MIGRATION}

Lymphocyte migration can be mediated by the formation of integrin-mediated adhesions (focal contacts) at the leading edge, which are translocated rearward resulting in forward motility (Smith et al. 2005). In fibroblasts and epithelial cells, the focal contacts move rearward and mature into larger structures called focal adhesions (Vogel and Sheetz 2006). In most cells, the highest concentration of focal adhesions are associated with the cellular actin structure known as the lamella, a structure found just behind the lamellopodia (Ponti et al. 2004; Ponti et al. 2005). Although the term focal adhesions is not widely used by T-cell biologists, integrin clusters are abundant within the pSMAC, a structure potentially analogous to the lamellae (Grakoui et al. 1999) (Fig. 1). The uropod contains the microtubule-organizing center (MTOC), and is also enriched in myosin IIA (Jacobelli et al. 2004; Morin et al. 2008). Myosin mediated contraction of the uropod is important for de-adhesion during motility (Jay et al. 1995). The positioning of the MTOC in the rear of the cell in lymphocytes is unusual because studies of most migrating cells, which are often much larger than lymphocytes, usually results in the positioning of the MTOC just behind the leading edge (Anderson et al. 1982; Gotlieb et al. 1981). Because the MTOC is the cytoplasmic center of the cell, its positioning to the rear of migrating lymphocytes may reflect packaging efficiency in the smaller cell and allow the lymphocyte extra flexibility in squeezing through endothelium and tightly packed tissue spaces.

\section{POLARITY AND ANTIGEN RECOGNITION}

The MTOC has long been used as a marker of changes in T-cell polarization in response to antigen stimulation. Its movement to the contact is thought to play a critical role in allowing directed secretion of cytotoxic granules by killer lymphocytes (Beal et al. 2009; Geiger et al. 1982; Kupfer et al. 1983; Stinchcombe et al. 2006). After a stable contact is formed, the uropod disassembles and the MTOC moves to a new position between the nucleus and the contact surface (Kupfer et al. 1983; Stinchcombe et al. 2006). With the MTOC in this position, the axis of polarity is now toward the antigen presenting cell surface, with the motility apparatus configured to sustain contact over many minutes to hours.

The exact mechanism of how the MTOC is reoriented is unclear but recent data suggest that one important factor is di-acyl-glycerol (DAG) (Quann et al. 2009). DAG is generated during the hydrolysis of PIP2 by the activation of phospholipase C. Because DAG is confined to the plasma membrane, and has a relatively short half-life, the localized production of DAG at the IS marks the position of activated receptors at the contact membrane. Localization of PLC- $\gamma$ to the IS allows for the local production of DAG and presumably the recruitment of proteins with DAG binding C1 domains. In fact, MTOC reorientation can be triggered solely by acute generation of DAG at one pole of a $T$ cell (Quann et al. 2009).

Movement of microtubules generally requires microtubule motors. Microtubules have a fast growing "plus" end and a slower growing "minus" end. The minus end is associated with the MTOC and the plus end is pointed at the plasma membrane. Two groups have shown that the minus end motor protein, dynein, is recruited to the immunological synapse and is required for MTOC repolarization (Combs et al. 2006; Martin-Cofreces et al. 2008). Dynein may be recruited to the IS by binding to the 
scaffold protein ADAP, suggesting that dynein recruitment may be linked to the activation of integrins (Combs et al. 2006; Peterson et al. 2001). Dynein may induce the reorientation of the MTOC by pulling microtubules and the MTOC toward the immunological synapse. The proximity of the MTOC to the contact surface may be critical as Griffiths and coworkers have shown that in CTLs, direct contact of the MTOC with the IS is critical for cytolytic granule release because the process appears to only depend on +end-directed motors (Stinchcombe et al. 2006).

\section{PAR PROTEINS AND THE GENETICS OF POLARITY}

The partition defective or Par proteins were originally identified based on their role in asymmetric cell division of the Caenorhabditis elegans oocyte (Kemphues et al. 1988). More recently, they have been shown to play important roles in directing the polarity of epithelial cells (Joberty et al. 2000; Lin et al. 2000). The role of Par proteins in regulating polarity in T lymphocytes is largely unknown. There are six distinct Par proteins spanning four structural families. Two of the proteins, Par1 and Par4 are serine/threonine kinases. Par3 and Par6 are PDZ domain containing adapter proteins. Par2 is a RING finger protein and Par 5 is a 14-3-3 protein. Another serine-threonine kinase, an atypical protein kinase $\mathrm{C}, \mathrm{PKC}-\zeta$, interacts with the Par proteins and plays an important role in polarity.

Typically, the Par3 and Par6s form a complex with PKC- $\zeta$. In the C. elegans oocyte, Par3/6/ $\mathrm{PKC}-\zeta$ are associated with the anterior portion of the cell whereas Par1 localizes to the posterior pole (Kemphues et al. 1988). In epithelial cells, the Par3/6/PKC- $\zeta$ complex is associated with apical junctional complexes (Joberty et al. 2000; Lin et al. 2000), whereas Par1 is associated with the basolateral surface (Bohm et al. 1997). The mutually exclusive localization of the Par3 complex and Parl is thought to be mediated by $\mathrm{PKC}-\zeta$ phosphorylation of Par 1 resulting in 14-3-3 binding and subsequent removal of Par1 from Par3/6/PKC- $\zeta$ containing membranes (Suzuki et al. 2004).
In T cells, the role of the Par proteins is less clear. Par3 moves to the synapse whereas PKC- $\zeta$ apparently localizes to the pole of the cell distal to the synapse (Ludford-Menting et al. 2005). Par1 appears to relocate to the synapse where it is phosphorylated by $\mathrm{PKC}-\zeta$ and resides in the cytoplasm below the synapse (Lin et al. 2009). Par1 appears to be important for polarization because expression of a dominant negative form of Par1 can block MTOC polarization (Lin et al. 2009). Interestingly, knockout mice lacking one of the Par1 isoforms, Par1b (EMK) develop autoimmunity suggesting that Par1 and cell polarity may play an important role in immunoregulation (Hurov et al. 2001).

Another important class of polarity proteins are PDZ containing adapter proteins known as Scribble (Scrb), Disc Large (Dlg), and Lethal Giant Larva (Lgl). In epithelial cells, these three molecules appear to interact with each other, are required for apical/basal polarity, and are localized to the basal/lateral membrane (Qin et al. 2005). Early in synapse formation, Scrb and Dlg are recruited to the synapse but at later time-points, they end up localized to the membrane distal to the synapse (Ludford-Menting et al. 2005). Inhibition of Scrb expression blocks motility and conjugate formation but the mechanism is not known.

\section{Forming the Bullseye}

The bullseye pattern with the integrins, forming the outer ring surrounding the TCR that is concentrated in a central spot, is a highly recognized feature of the T-cell immunological synapse (Grakoui et al. 1999; Monks et al. 1998). Although this pattern does not appear to be a universal feature of immunological synapses (Brossard et al. 2005; Tseng et al. 2008), its distinct structure has served as a central focus of researchers in the field.

Recent progress in understanding the formation of the immunological synapse has been facilitated by the use of artificial planar lipid bilayers as well as a method of imaging known as total interference reflection microscopy (TIRFM) (Campi et al. 2005; Yokosuka et al. 2005). The incorporation of mobile ligands into 
lipid bilayers has allowed the reorganization of surface receptors during $\mathrm{T}$-cell activation to be visualized in real-time and with optimal resolution (Grakoui et al. 1999). In contrast, the reorganization of TCRs and integrins into pSMACs and cSMACs does not occur in systems using antibody coated glass (Bunnell et al. 2002; Douglass and Vale 2005). In addition, the highly sensitive TIRFM method allows individual molecules or small clusters of molecules to be imaged (Varma et al. 2006; Yokosuka et al. 2005). These studies show that new engagement of antigen by the TCR first takes place in an area outside of the pSMAC, known as the distal-SMAC or dSMAC.

\section{MICROCLUSTERS IN THE SYNAPSE}

Antigen induces the formation of small TCR microclusters on the order of 11-17 TCRs per microcluster (Varma et al. 2006). Although the TCR microclusters can also include molecules like CD2, CD4, CD8, and CD28, they exclude the tyrosine phosphatase CD45 (Varma et al. 2006; Yokosuka et al. 2008), a step that may be important for TCR triggering (Choudhuri et al. 2005). Interestingly, LFA-1 also forms microclusters in the dSMAC that are segregated from the TCR microclusters (Kaizuka et al. 2007). Although both TCR and LFA-1 microclusters then move centripetally through the pSMAC (Kaizuka et al. 2007), experiments using barriers placed in the bilayer (Mossman et al. 2005) show that the TCR microclusters are more stable than the more labile LFA-1 clusters. The TCR microclusters have a limited capacity to navigate around small obstacles in their path to the cSMAC, supporting a "frictional coupling" model between TCR and the actin cytoskeleton (DeMond et al. 2008). The TCR microclusters are then translocated into the actin poor cSMAC by a TSG101 dependent mechanism (Vardhana 2010), whereas free ICAM-1 in the bilayer appears to be poorly able to diffuse into the cSMAC, perhaps limiting accumulation of LFA-1-ICAM-1 complexes. It should be noted that TCR microclusters have yet to be visualized in the IS of bona fide T cell/ APC conjugates.
The centripetal movement of TCR and LFA-1 microclusters is actin dependent and parallels the retrograde actin flow that occurs during cell spreading and migration (Kaizuka et al. 2007; Varma et al. 2006). At the extreme periphery of the contact area in the dSMAC, actin filaments grow rapidly because of the high concentration of Arp2/3 and cofilin, which increase the number of growing filaments (Sims et al. 2007). Behind the dSMAC, in the pSMAC, the actin filaments are anchored to clusters of integrins like LFA-1 through adapters such as talin (Monks et al. 1998). This anchoring impedes the free centripetal movement of filaments but contributes to the force of the rapidly growing actin filaments on the plasma membrane $(\mathrm{Hu}$ et al. 2007). In the pSMAC, the retrograde actin flow is linked to substrate attached integrins and the speed of cell movement is determined by the slippage between the integrin mediated anchors as well as the activity of myosin IIa, which contracts and integrates the actin network (Ilani et al. 2009; Jacobelli et al. 2004; Morin et al. 2008). The LFA1 ligand ICAM-1 can slide across the membrane of the antigen-presenting cell allowing for the formation of the rings of LFA- 1 and ICAM-1 on the T cell and APC respectively (Carrasco et al. 2004).

Because of the absence of actin in the cSMAC, it was initially proposed that the ability of the TCR to accumulate in the cSMAC might be based on an unknown mechanism that was actin independent. Although the formation of both TCR and LFA-1 microclusters requires actin, TCR, and not LFA-1 microclusters become F-actin-independent suggesting a distinct transport mechanism (Bunnell et al. 2002; Kaizuka et al. 2007; Sims et al. 2007; Varma et al. 2006). In the case of the actin dependent LFA1 clusters, one idea is that they disperse when they reach F-actin free cSMAC (Kaizuka et al. 2007). This model postulates that the cSMAC is a container that collects cargo that falls off the actinomyosin conveyor belt as it reaches the border of the pSMAC and the cSMAC. In this model, because TCR clusters are actin independent, they can persist after falling into the cSMAC. This model is supported by recent evidence showing that LFA-1 cross-linking 
antibodies promote the transport of LFA-1ICAM-1 complexes into the cSMAC (Hartman et al. 2009). Although the authors interpreted this result in terms of cluster size and frictional coupling to centripetally moving F-actin, it is also possible that the cross-linking antibodies stabilize the LFA-1 clusters and allows the clusters to persist in the F-actin poor CSMAC.

It is also possible to explain the inability of LFA-1 clusters to penetrate the border of the cSMAC and the PSMAC based on the size mismatches between the TCR and LFA-1 (Springer, 1990). The TCR bound to the MHC is estimated to span a distance of about $15 \mathrm{~nm}$, whereas the distance spanned by the LFA-1/ICAM pair is estimated at over $40 \mathrm{~nm}$ (Springer 1990). Consistent with this, LFA- 1 and TCR microclusters are segregated laterally as soon as they form and the accumulation of TCR microclusters in cSMAC might form a tight surface that is not penetrable by the LFA-1/ICAM-1 pair (Kaizuka et al. 2007; Qi et al. 2001; Shaw and Dustin 1997).

Studies of the localization of PKC- $\theta$ (Monks et al. 1998) and CD28 in the synapse suggest that the cSMAC may have two distinct compartments. CD28 and PKC- $\theta$ are strongly colocalized and high-resolution imaging shows lateral segregation from most of the TCR accumulated in the core of the cSMAC (Yokosuka et al. 2008). This result splits the cSMAC into two distinct compartments. The localization of a signaling molecule in a distinct outer zone suggests that the cSMAC may be divided into two functional domains, with a signaling component localized in the area just inside the pSMAC (Yokosuka et al. 2008). These dynamic central compartments may account for signaling activity detected in the cSMAC in CD4 and CD8 T cells (Beal et al. 2009; Cemerski et al. 2008; Jenkins et al. 2009) and is discussed in more detail in the next section of the article.

\section{Function of the Synapse}

The function of the immunological synapse has been a controversial area and confused by different definitions of the immunological synapse (Davis and Dustin 2004). If the term is meant to define stable contact surface between $\mathrm{T}$ cell and APC (Dustin et al. 1998; Mempel et al. 2004), then the question is what is the role of sustained contact between these two cells. For others, the term refers to the reorganization of the contact area into the pSMAC and the cSMAC (Monks et al. 1998). In this case, the question refers mainly to the function of the CSMAC versus the pSMAC Lastly, the term immunological synapse sometimes refers to the contact surface between a CTL and a target cell (Stinchcombe et al. 2006). In this case, the issue is what the role of the PSMAC and cSMAC are in cytolytic killing. Regardless, each of these areas has been an active area of research.

\section{THE SYNAPSE AND STABLE CONTACTS}

The requirement for stable contacts for T-cell activation is an issue that has been largely resolved over the last $5 \mathrm{yr}$, but the biological implications remain controversial. Lanzavecchia initially proposed that naïve $\mathrm{T}$ cells require many hours of stable contact before becoming committed to divide (Iezzi et al. 1998). Continuous imaging of $\mathrm{T}$ cells interacting with dendritic cells in a three-dimensional collagen matrix showed that $\mathrm{T}$ cells can, however, proliferate after many transient interactions (Gunzer et al. 2000). In vivo imaging showed that $T$ cells transition from transient interactions to stable contacts that persists for many hours (Mempel et al. 2004; Shakhar et al. 2005). Experiments with ICAM-1 deficient mice and delivery of antigen to all dendritic cells confirmed that stable contacts are not required for T-cell proliferation and early cytokine release, but suggested that they are required for T-cell memory (Scholer et al. 2008). It remains possible that stable contacts between T cells and DC will be more important when antigen is presented on more physiological subpopulations of dendritic cells (Dustin 2008).

\section{THE FUNCTION OF THE PSMAC AND CSMAC}

What is the role of the cSMAC and pSMAC? Because the cSMAC is notable for high concentrations of TCRs, it was initially postulated to 
function as a site of TCR signaling by facilitating sustained recognition of low-affinity ligands for the TCR (Grakoui et al. 1999; Monks et al. 1998). But it is clear now that formation of the cSMAC is not necessary to initiate signaling, rather, as discussed previously, TCRs form small microclusters in the pSMAC that are associated with the initiation of signaling (Campi et al. 2005; Yokosuka et al. 2005). These studies also suggest that continuous microcluster formation is required for sustained signaling because microcluster signaling appears to attenuate as the microclusters reach the cSMAC (Campi et al. 2005; Yokosuka et al. 2005). One possible function of the cSMAC is that it may function to terminate signaling.

Initially, because TCRs are concentrated in the cSMAC, it was assumed that signaling by the TCR would occur mainly in the cSMAC (Grakoui et al. 1999; Monks et al. 1998). Staining the IS with phosphotyrosine antibodies suggested surprisingly that signaling was low in the cSMAC compared to the PSMAC (Lee et al. 2002; Lee et al. 2003). Further experimentation showed that this issue was complicated as the intensity of phosphotyrosine staining varied depending on the quality of the agonist. Strong peptides tended to result in less phosphotyrosine staining in the cSMAC whereas weak peptides tended to show more phosphotyrosine staining in the cSMAC (Cemerski et al. 2008). These experiments are complicated by the fact that the efficiency of cSMAC formation is also related to agonist quality, with stronger peptides generating cSMACs with better efficiency (Grakoui et al. 1999; Monks et al. 1998).

Because the eventual fate of many TCRs that are triggered by agonist ligands is degradation in lysosomal compartments (Valitutti et al. 1997), one interpretation is that the cSMAC functions as a specific compartment to facilitate ubiquitination and degradation of the TCR (Lee et al. 2003; Lee et al. 2002). Strong antigenic peptides would both enhance the formation of the cSMAC and degradation of the T-cell receptor resulting in lower phosphotyrosine detection in the cSMAC. Because weak antigenic peptides might have less capacity to stimulate receptor degradation, their stimulation results in more persistent phosphotyrosine detection in the cSMAC. The observation that the cSMAC is rich in lysobisphosphatidic acid (Varma et al. 2006), a marker of multivesicular bodies, supports a role of the cSMAC in receptor degradation for strong peptides and further suggests that the cSMAC may function actively in the sorting process leading to lysosomal targeting of membrane proteins (Williams and Urbe 2007).

Combining a specific role for the cSMAC in receptor degradation with the microcluster data suggests that continuous and sustained signaling might require the continuous formation of new TCR microclusters in the periphery of the contact (Varma et al. 2006; Yokosuka et al. 2005). This model requires that the APC also have a continuous supply of antigenic pMHC complexes available at the periphery of the contact site for many hours. This model is consistent with much of the existing data with but also with the caveat that most of this data was generated with TIRFM on artificial lipid bilayers. If the model is correct, one important question to be resolved is why signaling occurs in the peripheral microclusters and not in the TCRs clustered in the cSMAC.

This model is, however, not consistent with the presence of phosphotyrosine staining in the cSMAC seen with weak agonist stimulation. One possible explanation is the recent data suggesting that the cSMAC has at least two functional domains. The finding that CD28 and PKC- $\theta$ are strongly colocalized and segregated from most of the TCR accumulated in the core of the cSMAC (Yokosuka et al. 2008). This result splits the cSMAC into two distinct compartments, one that is more dynamic and may remain active in signaling. Similar structures were detected in multifocal $\mathrm{T}$ cell-DC IS, in which CD80 and PKC- $\theta$ rich compartments were seen to be continuously dependent on dynamic TCR signaling (Tseng et al. 2008). These dynamic central compartments could account for signaling activity detected in the cSMAC in CD4 and CD8 T cells (Cemerski et al. 2008; Jenkins et al. 2009). 


\section{COMPUTATIONAL APPROACHES TO UNDERSTAND SYNAPSE FUNCTION}

Elucidating the signaling function of the synapse, and the cSMAC in particular, has proven to be difficult because there are many competing effects and components at play, which makes it difficult to intuit mechanisms from experimental observations of a few variables. Computational and theoretical models have been applied to complement experimental studies to help unravel the interplay of complex-competing effects in various aspects of T-cell signaling (Altan-Bonnet and Germain 2005; Cemerski et al. 2007; Chakraborty and Das 2010; Das et al. 2009; Goldstein et al. 2002; Lee et al. 2003). This is advantageous because computational models can keep track of the outcomes of different mechanistic hypotheses for each signaling component and determine whether each hypothesis yields results consistent with experimental observations. Because of the complex nature of the interactions, some hypotheses that seem plausible intuitively may be inconsistent with experimental observations, and these can be ruled out by computational modeling. The computational models can also be used to design experiments that can sensitively discriminate between competing plausible hypotheses, which are consistent with all known experimental facts. Such complementary computational and experimental investigations have aided the quest to understand the formation of and signaling in the immunological synapse.

Shortly after the first report of the low levels of active signaling molecules in the cSMAC (Lee et al. 2002), a computational model was formulated (Lee et al. 2003) to try and shed light on the mechanistic origin of this observation. The computational model placed the TCR and pMHC molecules on two apposed surfaces, and simulated their binding to each other when they were within a certain distance of each other. A threshold level of signaling was used to initiate forces that led to cSMAC formation. The model also simulated the phosphorylation and dephosphorylation of the ITAMs as a function of the binding kinetics of the TCR and pMHC, allowing for fully or partially phosphorylated
ITAMs. As the model was developed, it incorporated aspects of ubiquitination and receptor down-regulation and degradation (Liu et al. 2000; Naramura et al. 2002). Because biochemical reactions are stochastic events, the computational models explicitly considered these stochastic fluctuations. Importantly, the formation of the cSMAC could be turned on or off at will in the simulations, thus allowing one to assess the potential role of cSMAC formation on TCR signaling under different conditions.

Computational studies indicated that the overall amount of signaling as well as its ability to be detected could be affected by cSMAC formation. The model suggested that for strong agonists, the long half-life of the TCR/pMHC complex would allow for efficient TCR triggering before being transported to the CSMAC, consistent with what was subsequently seen in the TCR microcluster studies (Varma et al. 2006). The model also showed that the process of concentrating receptors in the center of the contact area (cSMAC) would promote receptor degradation by helping to concentrate both phosphorylated TCRs and ubiquitination agents in the same local area. The model, however, also warned experimentalists that low levels of signaling intermediates in the cSMAC might actually reflect rapid degradation of active signaling components and not low levels of signaling. Supporting this idea, experiments using agents to slow receptor degradation resulted in increased detection of phosphotyrosine in the cSMAC (Cemerski et al. 2008).

The computational studies also suggested other implications for the effects of cSMAC formation on signaling. The calculations predicted that assuming that the cSMAC could form, it could enhance signaling induced by weak ligands. Although weak ligands would result in only partially phosphorylated receptors prior to cSMAC formation, concentrating them in the cSMAC along with other signaling components would promote more complete phosphorylation because the higher concentration of receptors and ligands would enhance receptor occupancy. Only subsequently, would the cSMAC enhance degradation. Using manuipulations that enhance cSMAC formation, it was 
shown that CSMAC formation could enhance signaling by weak ligands (Cemerski et al. 2007). Although the efficiency of cSMAC formation is related to antigen quality, suggesting that cSMACs do not form in the presence of weak ligands, evidence suggests that nonTCR stimuli can allow for CSMAC formation in the absence of antigen. For example, the cytokine IL-12 and the expression of stress activated ligands for NKG2D can lower the threshold for CSMAC formation. (Markiewicz et al. 2005; Somersalo et al. 2004). It therefore remains a possibility that the CSMAC could serve to enhance signaling by weak ligands.

In spite of these insights, it should be noted that the computational models have not yet incorporated some of the new details that are emerging from the high-resolution TIRF microscopy studies. For example, the different compartments within the cSMAC have not yet been considered in the computational models. Combining models for microcluster formation, transport and SMAC formation explicitly with models for signaling will provide a pathway for quantitatively testing our understanding of how these events are coordinated. Toward this end, adapting and further advancing models of the coarse-grained structures that form during immunological synapse formation may be useful (Burroughs and Wulfing 2002; Qi et al. 2001; Weikl and Lipowsky 2004). The ability of such physical models to relate patterns to functional outcomes likely point to important physical processes linking membrane bending fluctuations, chemical kinetics, molecular topology and cytoskeletal transport, processes also likely to operate at the level of microcluster formation. Because the rules for microcluster formation are poorly understood simulations should play an important role in testing hypotheses.

\section{THE IMMUNOLOGICAL SYNAPSE AND THE KISS OF DEATH}

Lastly, formation of the cSMAC and pSMAC may be important for cytolytic killing. In this case, it has been postulated that the pSMAC functions as a "sealing ring" to prevent the escape of cytolytic agents secreted into the cSMAC during cytolysis as well as allow for the polarized secretion of cytokines and cytolytic agents into the cSMAC. Griffiths and coworkers showed using fluorescence and electron microscopy that in the CTL synapse with target cell, the cSMAC is divided into two zones (Stinchcombe et al. 2001). One zone is characterized by the concentration of receptors previously shown to be in the CSMAC, whereas the other zone functions as a space for the release of cytolytic granule contents. This suggests that the formation of a specific zone that is actin free and within the actin-rich pSMAC may be important for cytolytic killing.

In a recent series of studies, Sykulev and colleagues quantified the contribution of the pSMAC and granule delivery to the killing efficiency of cytotoxic T cells (Anikeeva et al. 2005; Beal et al. 2008; Beal et al. 2009). LFA-1 makes profound contributions to T-cell sensitivity and its interactions uniquely define the pSMAC. These effects were separated out in two studies with the conclusion that an intact pSMAC increases the efficiency of killing by threefold (Beal et al. 2008). The rate of granule exocytosis to the target cell is controlled by efficient granule delivery to the CSMAC. This parameter was controlled in turn by the intensity of signaling from the synapse and appears to account for a 30 -fold component of the difference between fast killing $\mathrm{CD}^{+}$and slow killing $\mathrm{CD} 4^{+} \mathrm{CTL}$ (Beal et al. 2009). Similar results were obtained with a $\mathrm{CD} 8^{+} \mathrm{T}$ cell system in which altered peptide ligands with weaker signals were less able to recruit granules to the MTOC at the CSMAC (Jenkins et al. 2009). Thus, the pSMAC and cSMAC both make significant quantitative contributions to the efficiency of CTL, neither structure is absolutely required for killing of targets.

\section{REFERENCES}

Altan-Bonnet G, Germain RN. 2005. Modeling T cell antigen discrimination based on feedback control of digital ERK responses. PLoS Biol 3: e356.

Anderson DC, Wible LJ, Hughes BJ, Smith CW, Brinkley BR. 1982. Cytoplasmic microtubules in polymorphonuclear leukocytes: effects of chemotactic stimulation and colchicine. Cell 31: 719-729. 
Anikeeva N, Somersalo K, Sims TN, Thomas VK, Dustin ML, Sykulev Y. 2005. Distinct role of lymphocyte function-associated antigen-1 in mediating effective cytolytic activity by cytotoxic T lymphocytes. Proc Natl Acad Sci 102: 6437-6442.

Babbitt BP, Allen PM, Matsueda G, Haber E, Unanue ER. 1985. Binding of immunogenic peptides to Ia histocompatibility molecules. Nature 317: 359-361.

Barcia C, Thomas CE, Curtin JF, King GD, Wawrowsky K, Candolfi M, Xiong WD, Liu C, Kroeger K, Boyer O, et al. 2006. In vivo mature immunological synapses forming SMACs mediate clearance of virally infected astrocytes from the brain. J Exp Med 203: 2095-2107.

Beal AM, Anikeeva N, Varma R, Cameron TO, Norris PJ Dustin ML, Sykulev Y. 2008. Protein Kinase C\{theta\} Regulates stability of the peripheral adhesion ring junction and contributes to the sensitivity of target cell lysis by CTL. J Immunol 181: 4815-4824.

Beal AM, Anikeeva N, Varma R, Cameron TO, VasiliverShamis G, Norris PJ, Dustin ML, Sykulev Y. 2009. Kinetics of early $\mathrm{T}$ cell receptor signaling regulate the pathway of lytic granule delivery to the secretory domain. Immunity 31: 632-642.

Bohm H, Brinkmann V, Drab M, Henske A, Kurzchalia TV. 1997. Mammalian homologues of C. elegans PAR-1 are asymmetrically localized in epithelial cells and may influence their polarity. Curr Biol 7: 603-606.

Brossard C, Feuillet V, Schmitt A, Randriamampita C, Romao M, Raposo G, Trautmann A. 2005. Multifocal structure of the T cell-dendritic cell synapse. Eur J Immunol 35: 1741-1753.

Bunnell SC, Hong DI, Kardon JR, Yamazaki T, McGlade CJ, Barr VA, Samelson LE. 2002. T cell receptor ligation induces the formation of dynamically regulated signaling assemblies. J Cell Biol 158: 1263-1275.

Burroughs NJ, Wulfing C. 2002. Differential segregation in a cell-cell contact interface: the dynamics of the immunological synapse. Biophys J 83: 1784-1796.

Campi G, Varma R, Dustin ML. 2005. Actin and agonist MHC-peptide complex-dependent $\mathrm{T}$ cell receptor microclusters as scaffolds for signaling. J Exp Med 202: 1031-1036.

Carrasco YR, Fleire SJ, Cameron T, Dustin ML, Batista FD. 2004. LFA-1/ICAM-1 interaction lowers the threshold of B cell activation by facilitating B cell adhesion and synapse formation. Immunity 20: 589-599.

Celli S, Lemaitre F, Bousso P. 2007. Real-time manipulation of $\mathrm{T}$ cell-dendritic cell interactions in vivo reveals the importance of prolonged contacts for $\mathrm{CD} 4+\mathrm{T}$ cell activation. Immunity 27: 625-634.

Cemerski S, Das J, Giurisato E, Markiewicz MA, Allen PM, Chakraborty AK, Shaw AS. 2008. The balance between T cell receptor signaling and degradation at the center of the immunological synapse is determined by antigen quality. Immunity 29: 414-422.

Cemerski S, Das J, Locasale J, Arnold P, Giurisato E, Markiewicz MA, Fremont D, Allen PM, Chakraborty AK, Shaw AS. 2007. The stimulatory potency of T cell antigens is influenced by the formation of the immunological synapse. Immunity 26: 345-355.
Chakraborty AK, Das J. 2010. Pairing computation with experimentation: A powerful coupling for understanding T cell signalling. Nat Rev Immunol 10: 59-71.

Choudhuri K, Wiseman D, Brown MH, Gould K, van der Merwe PA. 2005. T-cell receptor triggering is critically dependent on the dimensions of its peptide-MHC ligand. Nature 436: 578-582.

Combs J, Kim SJ, Tan S, Ligon LA, Holzbaur EL, Kuhn J, Poenie M. 2006. Recruitment of dynein to the Jurkat immunological synapse. Proc Natl Acad Sci 103: 1488314888.

Das J, Kardar M, Chakraborty AK. 2009. Positive feedback regulation results in spatial clustering and fast spreading of active signaling molecules on a cell membrane. JChem Phys 130: 245102.

Davis DM, Dustin ML. 2004. What is the importance of the immunological synapse? Trends Immunol 25: 323-327.

DeMond AL, Mossman KD, Starr T, Dustin ML, Groves JT. 2008. T cell receptor microcluster transport through molecular mazes reveals mechanism of translocation. Biophys J 94: 3286-3292.

Douglass AD, Vale RD. 2005. Single-molecule microscopy reveals plasma membrane microdomains created by protein-protein networks that exclude or trap signaling molecules in T cells. Cell 121: 937-950.

Dustin ML. 2008. T-cell activation through immunological synapses and kinapses. Immunol Rev 221: 77-89.

Dustin ML, Springer TA. 1989. T-cell receptor cross-linking transiently stimulates adhesiveness through LFA-1. Nature 341: 619-624.

Dustin ML, Bromley SK, Kan Z, Peterson DA, Unanue ER. 1997. Antigen receptor engagement delivers a stop signal to migrating T lymphocytes. Proc Natl Acad Sci 94: 3909-3913.

Dustin ML, Olszowy MW, Holdorf AD, Li J, Bromley S, Desai N, Widder P, Rosenberger F, van der Merwe PA, Allen PM, et al. 1998. A novel adaptor protein orchestrates receptor patterning and cytoskeletal polarity in T-cell contacts. Cell 94: 667-677.

Geiger B, Rosen D, Berke G. 1982. Spatial relationships of microtubule-organizing centers and the contact area of cytotoxic T lymphocytes and target cells. J Cell Biol 95: 137-143.

Goldstein B, Faeder JR, Hlavacek WS, Blinov ML, Redondo A, Wofsy C. 2002. Modeling the early signaling events mediated by FcepsilonRI. Mol Immunol 38: 1213-1219.

Gotlieb AI, May LM, Subrahmanyan L, Kalnins VI. 1981. Distribution of microtubule organizing centers in migrating sheets of endothelial cells. J Cell Biol 91: 589-594.

Grakoui A, Bromley SK, Sumen C, Davis MM, Shaw AS, Allen PM, Dustin ML. 1999. The immunological synapse: A molecular machine controlling T cell activation. Science 285: 221-227.

Gunzer M, Schafer A, Borgmann S, Grabbe S, Zanker KS, Brocker EB, Kampgen E, Friedl P. 2000. Antigen presentation in extracellular matrix: interactions of $\mathrm{T}$ cells with dendritic cells are dynamic, short lived, and sequential. Immunity 13: 323-332. 
Hartman NC, Nye JA, Groves JT. 2009. Cluster size regulates protein sorting in the immunological synapse. Proc Natl Acad Sci 106: 12729-12734.

Henrickson SE, Mempel TR, Mazo IB, Liu B, Artyomov MN, Zheng H, Peixoto A, Flynn MP, Senman B, Junt T, et al. 2008. T cell sensing of antigen dose governs interactive behavior with dendritic cells and sets a threshold for T cell activation. Nat Immunol 9: 282-291.

Hirsch E, Katanaev VL, Garlanda C, Azzolino O, Pirola L, Silengo L, Sozzani S, Mantovani A, Altruda F, Wymann MP. 2000. Central role for G protein-coupled phosphoinositide 3-kinase $\gamma$ in inflammation. Science 287: 1049-1053.

Hu K, Ji L, Applegate KT, Danuser G, Waterman-Storer CM. 2007. Differential transmission of actin motion within focal adhesions. Science 315: 111-115.

Hurov JB, Stappenbeck TS, Zmasek CM, White LS, Ranganath SH, Russell JH, Chan AC, Murphy KM, Piwnica-Worms H. 2001. Immune system dysfunction and autoimmune disease in mice lacking Emk (Par-1) protein kinase. Mol Cell Biol 21: 3206-3219.

Huse M, Klein LO, Girvin AT, Faraj JM, Li QJ, Kuhns MS, Davis MM. 2007. Spatial and temporal dynamics of $T$ cell receptor signaling with a photoactivatable agonist. Immunity 27: 76-88.

Iezzi G, Karjalainen K, Lanzavecchia A. 1998. The duration of antigenic stimulation determines the fate of naive and effector T cells. Immunity 8: 89-95.

Ilani T, Vasiliver-Shamis G, Vardhana S, Bretscher A, Dustin ML. 2009. T cell antigen receptor signaling and immunological synapse stability require myosin IIA. Nat Immunol 10: $531-539$.

Irvine DJ, Purbhoo MA, Krogsgaard M, Davis MM. 2002. Direct observation of ligand recognition by $\mathrm{T}$ cells. Nature 419: 845-849.

Jacobelli J, Chmura SA, Buxton DB, Davis MM, Krummel MF. 2004. A single class II myosin modulates T cell motility and stopping, but not synapse formation. Nat Immunol 5: 531-538.

Jay PY, Pham PA, Wong SA, Elson EL. 1995. A mechanical function of myosin II in cell motility. J Cell Sci 108: 387-393.

Jenkins MR, Tsun A, Stinchcombe JC, Griffiths GM. 2009. The strength of $\mathrm{T}$ cell receptor signal controls the polarization of cytotoxic machinery to the immunological synapse. Immunity 31: 621-631.

Joberty G, Petersen C, Gao L, Macara IG. 2000. The cellpolarity protein Par6 links Par3 and atypical protein kinase C to Cdc42. Nat Cell Biol 2: 531-539.

Kaizuka Y, Douglass AD, Vardhana S, Dustin ML, Vale RD. 2009. The coreceptor CD2 uses plasma membrane microdomains to transduce signals in T cells. J Cell Biol 185: 521-534.

Kaizuka Y, Douglass AD, Varma R, Dustin ML, Vale RD. 2007. Mechanisms for segregating $T$ cell receptor and adhesion molecules during immunological synapse formation in Jurkat T cells. Proc Natl Acad Sci 104: 20296-20301.

Kemphues KJ, Priess JR, Morton DG, Cheng NS. 1988. Identification of genes required for cytoplasmic localization in early C. elegans embryos. Cell 52: 311-320.
Kupfer A, Dennert G, Singer SJ. 1983. Polarization of the Golgi apparatus and the microtubule-organizing center within cloned natural killer cells bound to their targets. Proc Natl Acad Sci 80: 7224-7228.

Lee KH, Dinner AR, Tu C, Campi G, Raychaudhuri S, Varma R, Sims TN, Burack WR, Wu H, Wang J, et al. 2003. The immunological synapse balances $\mathrm{T}$ cell receptor signaling and degradation. Science 302: 1218-1222.

Lee KH, Holdorf AD, Dustin ML, Chan AC, Allen PM, Shaw AS. 2002. T cell receptor signaling precedes immunological synapse formation. Science 295: 1539-1542.

Li Z, Jiang H, Xie W, Zhang Z, Smrcka AV, Wu D. 2000. Roles of PLC- $\beta 2$ and $-\beta 3$ and PI3K $\gamma$ in chemoattractantmediated signal transduction. Science 287: 1046-1049.

Lin D, Edwards AS, Fawcett JP, Mbamalu G, Scott JD, Pawson T. 2000. A mammalian PAR-3-PAR-6 complex implicated in Cdc42/Rac1 and aPKC signalling and cell polarity. Nat Cell Biol 2: 540-547.

Lin J, Hou KK, Piwnica-Worms H, Shaw AS. 2009. The polarity protein Par1b/EMK/MARK2 regulates $\mathrm{T}$ cell receptor-induced microtubule-organizing center polarization. J Immunol 183: 1215-1221.

Liu H, Rhodes M, Wiest DL, Vignali DA. 2000. On the dynamics of TCR:CD3 complex cell surface expression and downmodulation. Immunity 13: 665-675.

Ludford-Menting MJ, Oliaro J, Sacirbegovic F, Cheah ET, Pedersen N, Thomas SJ, Pasam A, Iazzolino R, Dow LE, Waterhouse NJ, et al. 2005. A network of PDZ-containing proteins regulates $\mathrm{T}$ cell polarity and morphology during migration and immunological synapse formation. Immunity 22: 737-748.

Markiewicz MA, Carayannopoulos LN, Naidenko OV, Matsui K, Burack WR, Wise EL, Fremont DH, Allen PM, Yokoyama WM, Colonna M, et al. 2005. Costimulation through NKG2D enhances murine CD8+ CTL function: Similarities and differences between NKG2D and CD28 costimulation. J Immunol 175: 2825-2833.

Martin-Cofreces NB, Robles-Valero J, Cabrero JR, Mittelbrunn M, Gordon-Alonso M, Sung CH, Alarcon B, Vazquez J, Sanchez-Madrid F. 2008. MTOC translocation modulates IS formation and controls sustained T cell signaling. J Cell Biol 182: 951-962.

McGavern DB, Christen U, Oldstone MB. 2002. Molecular anatomy of antigen-specific $\mathrm{CD} 8(+) \mathrm{T}$ cell engagement and synapse formation in vivo. Nat Immunol 3: 918-925.

Mempel TR, Henrickson SE, Von Andrian UH. 2004. T-cell priming by dendritic cells in lymph nodes occurs in three distinct phases. Nature 427: 154-159.

Miller MJ, Hejazi AS, Wei SH, Cahalan MD, Parker I. 2004. T cell repertoire scanning is promoted by dynamic dendritic cell behavior and random $\mathrm{T}$ cell motility in the lymph node. Proc Natl Acad Sci 101: 998-1003.

Monks CR, Freiberg BA, Kupfer H, Sciaky N, Kupfer A. 1998. Three-dimensional segregation of supramolecular activation clusters in T cells. Nature 395: 82-86.

Morin NA, Oakes PW, Hyun YM, Lee D, Chin EY, King MR, Springer TA, Shimaoka M, Tang JX, Reichner JS, et al. 2008. Nonmuscle myosin heavy chain IIA mediates integrin LFA-1 de-adhesion during T lymphocyte migration. J Exp Med 205: 195-205. 
Mossman KD, Campi G, Groves JT, Dustin ML. 2005. Altered TCR signaling from geometrically repatterned immunological synapses. Science 310: 1191-1193.

Naramura M, Jang IK, Kole H, Huang F, Haines D, Gu H. 2002. c-Cbl and Cbl-b regulate $\mathrm{T}$ cell responsiveness by promoting ligand-induced TCR down-modulation. Nat Immunol 3: 1192-1199.

Negulescu PA, Krasieva TB, Khan A, Kerschbaum HH, Cahalan MD. 1996. Polarity of T cell shape, motility, and sensitivity to antigen. Immunity 4: 421-430.

Niggli V, Keller H. 1997. The phosphatidylinositol 3-kinase inhibitor wortmannin markedly reduces chemotactic peptide-induced locomotion and increases in cytoskeletal actin in human neutrophils. Eur J Pharmacol 335: 43-52.

Norcross MA. 1984. A synaptic basis for T-lymphocyte activation. Ann Immunol (Paris) 135D: 113-134.

Peterson EJ, Woods ML, Dmowski SA, Derimanov G, Jordan MS, Wu JN, Myung PS, Liu QH, Pribila JT, Freedman BD, et al. 2001. Coupling of the TCR to integrin activation by Slap-130/Fyb. Science 293: 2263-2265.

Ponti A, Machacek M, Gupton SL, Waterman-Storer CM, Danuser G. 2004. Two distinct actin networks drive the protrusion of migrating cells. Science 305: 1782-1786.

Ponti A, Matov A, Adams M, Gupton S, Waterman-Storer CM, Danuser G. 2005. Periodic patterns of actin turnover in lamellipodia and lamellae of migrating epithelial cells analyzed by quantitative Fluorescent Speckle Microscopy. Biophys J 89: 3456-3469.

Purtic B, Pitcher LA, van Oers NS, Wulfing C. 2005. T cell receptor (TCR) clustering in the immunological synapse integrates TCR and costimulatory signaling in selected T cells. Proc Natl Acad Sci 102: 2904-2909.

Qi SY, Groves JT, Chakraborty AK. 2001. Synaptic pattern formation during cellular recognition. Proc Natl Acad Sci 98: 6548-6553.

Qin Y, Capaldo C, Gumbiner BM, Macara IG. 2005. The mammalian Scribble polarity protein regulates epithelial cell adhesion and migration through E-cadherin. J Cell Biol 171: 1061-1071.

Quann EJ, Merino E, Furuta T, Huse M. 2009. Localized diacylglycerol drives the polarization of the microtubule-organizing center in $\mathrm{T}$ cells. Nat Immunol 10: 627-635.

Reichert P, Reinhardt RL, Ingulli E, Jenkins MK. 2001. Cutting edge: In vivo identification of TCR redistribution and polarized IL-2 production by naive CD4 T cells. J Immunol 166: 4278-4281.

Sanchez-Madrid F, Serrador JM. 2009. Bringing up the rear: defining the roles of the uropod. Nat Rev Mol Cell Biol 10: 353-359.

Scholer A, Hugues S, Boissonnas A, Fetler L, Amigorena S. 2008. Intercellular adhesion molecule-1-dependent stable interactions between $\mathrm{T}$ cells and dendritic cells determine CD8 + T cell memory. Immunity 28: 258-270.

Shakhar G, Lindquist RL, Skokos D, Dudziak D, Huang JH, Nussenzweig MC, Dustin ML. 2005. Stable T celldendritic cell interactions precede the development of both tolerance and immunity in vivo. Nat Immunol 6: 707-714.
Shaw AS, Dustin ML. 1997. Making the T cell receptor go the distance: A topological view of $\mathrm{T}$ cell activation. Immunity 6: 361-369.

Sims TN, Soos TJ, Xenias HS, Dubin-Thaler B, Hofman JM, Waite JC, Cameron TO, Thomas VK, Varma R, Wiggins $\mathrm{CH}$, et al. 2007. Opposing effects of PKC $\theta$ and WASp on symmetry breaking and relocation of the immunological synapse. Cell 129: 773-785.

Skokos D, Shakhar G, Varma R, Waite JC, Cameron TO, Lindquist RL, Schwickert T, Nussenzweig MC, Dustin ML. 2007. Peptide-MHC potency governs dynamic interactions between $\mathrm{T}$ cells and dendritic cells in lymph nodes. Nat Immunol 8: 835-844.

Smith A, Carrasco YR, Stanley P, Kieffer N, Batista FD, Hogg N. 2005. A talin-dependent LFA- 1 focal zone is formed by rapidly migrating $\mathrm{T}$ lymphocytes. J Cell Biol 170: 141-151.

Somersalo K, Anikeeva N, Sims TN, Thomas VK, Strong RK, Spies T, Lebedeva T, Sykulev Y, Dustin ML. 2004. Cytotoxic T lymphocytes form an antigen-independent ring junction. J Clin Invest 113: 49-57.

Springer TA. 1990. Adhesion receptors of the immune system. Nature 346: 425-434.

Stinchcombe JC, Bossi G, Booth S, Griffiths GM. 2001. The immunological synapse of CTL contains a secretory domain and membrane bridges. Immunity 15: 751-761.

Stinchcombe JC, Majorovits E, Bossi G, Fuller S, Griffiths GM. 2006. Centrosome polarization delivers secretory granules to the immunological synapse. Nature 443: $462-465$.

Suzuki A, Hirata M, Kamimura K, Maniwa R, Yamanaka T, Mizuno K, Kishikawa M, Hirose H, Amano Y., Izumi N, et al. 2004. aPKC acts upstream of PAR-1b in both the establishment and maintenance of mammalian epithelial polarity. Curr Biol 14: 1425-1435.

Tseng SY, Waite JC, Liu M, Vardhana S, Dustin ML. 2008. T cell-dendritic cell immunological synapses contain TCR-dependent CD28-CD80 clusters that recruit protein kinase Ctheta. J Immunol 181: 4852-4863.

Valitutti S, Muller S, Salio M, Lanzavecchia A. 1997. Degradation of T cell receptor (TCR)-CD3- - complexes after antigenic stimulation. J Exp Med 185: 1859-1864.

Vardhana S, Choudhuri K, Varma R, Dustin ML. 2010. Essential role of ubiquitin and TSG101 protein in formation and function of the central supramolecular activation cluster. Immunity 32: 531-540.

Varma R, Campi G, Yokosuka T, Saito T, Dustin ML. 2006. T cell receptor-proximal signals are sustained in peripheral microclusters and terminated in the central supramolecular activation cluster. Immunity 25: 117-127.

Vogel V, Sheetz M. 2006. Local force and geometry sensing regulate cell functions. Nat Rev Mol Cell Biol 7: 265-275.

Weikl TR, Lipowsky R. 2004. Pattern formation during T-cell adhesion. Biophys J 87: 3665-3678.

Wiedemann A, Muller S, Favier B, Penna D, Guiraud M, Delmas C, Champagne E, Valitutti S. 2005. T-cell activation is accompanied by an ubiquitination process occurring at the immunological synapse. Immunol Lett 98: $57-61$.

Williams RL, Urbe S. 2007. The emerging shape of the ESCRT machinery. Nat Rev Mol Cell Biol 8: 355-368. 
M.L. Dustin, A.K. Chakraborty, and A.S. Shaw

Woolf E, Grigorova I, Sagiv A, Grabovsky V, Feigelson SW, Shulman Z, Hartmann T, Sixt M, Cyster JG, Alon R. 2007. Lymph node chemokines promote sustained $\mathrm{T}$ lymphocyte motility without triggering stable integrin adhesiveness in the absence of shear forces. Nat Immunol 8: $1076-1085$.

Yokosuka T, Kobayashi W, Sakata-Sogawa K, Takamatsu M, Hashimoto-Tane A, Dustin ML, Tokunaga M, Saito T.
2008. Spatiotemporal regulation of $\mathrm{T}$ cell costimulation by TCR-CD28 microclusters and protein kinase C Өtranslocation. Immunity 29: 589-601.

Yokosuka T, Sakata-Sogawa K, Kobayashi W, Hiroshima M, Hashimoto-Tane A, Tokunaga M, Dustin ML, Saito T. 2005. Newly generated T cell receptor microclusters initiate and sustain $\mathrm{T}$ cell activation by recruitment of Zap70 and SLP-76. Nat Immunol 6: 1253-1262. 


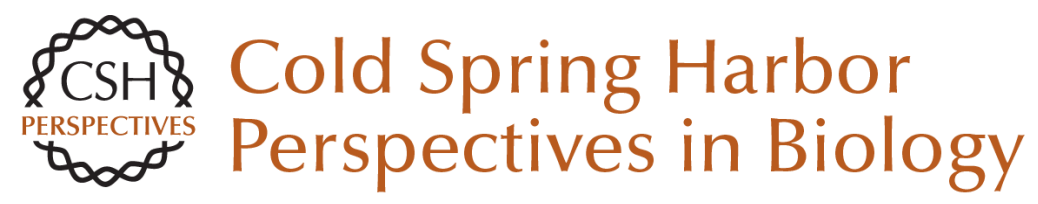

\title{
Understanding the Structure and Function of the Immunological Synapse
}

\author{
Michael L. Dustin, Arup K. Chakraborty and Andrey S. Shaw
}

Cold Spring Harb Perspect Biol 2010; doi: 10.1101/cshperspect.a002311 originally published online September 15, 2010

\section{Subject Collection Immunoreceptor Signaling}

The Coordination of T-cell Function by

Serine/Threonine Kinases

David Finlay and Doreen Cantrell

ITAM-mediated Signaling by the T-Cell Antigen

Receptor

Paul E. Love and Sandra M. Hayes

Coordination of Receptor Signaling in Multiple Hematopoietic Cell Lineages by the Adaptor

Protein SLP-76

Martha S. Jordan and Gary A. Koretzky

The Cytoskeleton Coordinates the Early Events of B-cell Activation

Naomi E. Harwood and Facundo D. Batista

An Enigmatic Tail of CD28 Signaling

Jonathan S. Boomer and Jonathan M. Green

Mediation of T-Cell Activation by Actin

Meshworks

Peter Beemiller and Matthew F. Krummel
Perspectives for Computer Modeling in the Study

of T Cell Activation Jesse Coward, Ronald N. Germain and Grégoire Altan-Bonnet

Structural Biology of the T-cell Receptor: Insights into Receptor Assembly, Ligand Recognition, and Initiation of Signaling

Kai W. Wucherpfennig, Etienne Gagnon, Melissa J. Call, et al.

Src-family and Syk Kinases in Activating and Inhibitory Pathways in Innate Immune Cells:

Signaling Cross Talk Clifford A. Lowell

The LAT Story: A Tale of Cooperativity,

Coordination, and Choreography Lakshmi Balagopalan, Nathan P. Coussens, Eilon Sherman, et al.

Antigen Receptor Signaling to NF-KB via

CARMA1, BCL10, and MALT1 Margot Thome, Jean Enno Charton, Christiane Pelzer, et al.

It's All About Change: The Antigen-driven Initiation of B-Cell Receptor Signaling Wanli Liu, Hae Won Sohn, Pavel Tolar, et al.

For additional articles in this collection, see http://cshperspectives.cshlp.org/cgi/collection/

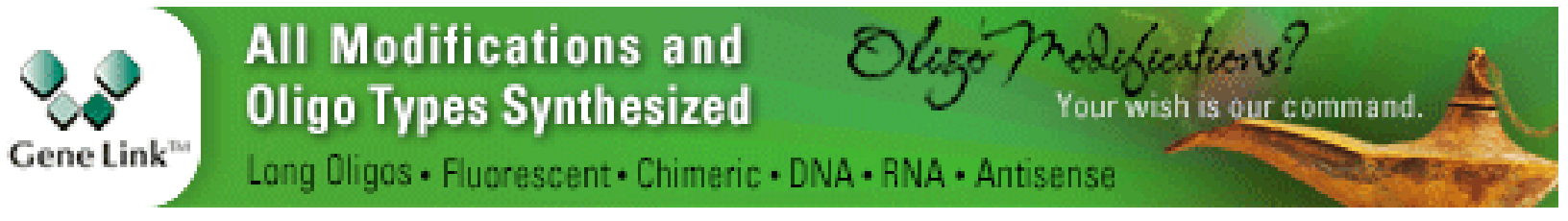


T-Cell Signaling Regulated by the Tec Family

Kinase, Itk

Amy H. Andreotti, Pamela L. Schwartzberg, Raji E. Joseph, et al.

Lipid Signaling in T-Cell Development and

Function

Yina H. Huang and Karsten Sauer
ZAP-70: An Essential Kinase in T-cell Signaling Haopeng Wang, Theresa A. Kadlecek, Byron B. Au-Yeung, et al.

Understanding the Structure and Function of the Immunological Synapse

Michael L. Dustin, Arup K. Chakraborty and Andrey S. Shaw

For additional articles in this collection, see http://cshperspectives.cshlp.org/cgi/collection/

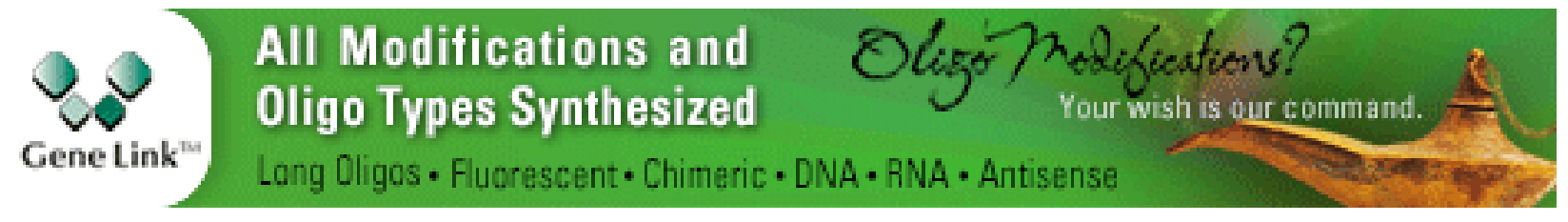

Copyright @ 2010 Cold Spring Harbor Laboratory Press; all rights reserved 